\title{
ELECTRON DENSITY AND NITROGEN ABUNDANCE FROM FIR LINES
}

\author{
R.H. RUBIN, S.W.J. COLGAN, E.F. ERICKSON, M.R. HAAS, S.D. LORD and J.P. SIMPSON \\ NASA Ames Research Center, M.S. 245 - 6, Moffett Field, CA 94035, USA
}

In order to study the physical properties of nebulae and determine their elemental abundances, it is important to observe lines from many different ionic species. Such studies have been enhanced in recent years with the measurement of lines in the far-infrared (FIR). The [N III] $57 \mu \mathrm{m}$ line provides a way to assess the $\mathrm{N}^{++}$abundance - which is not readily done from any other spectral region. Recent detection of the [N II] 122 and $205 \mu$ m lines provides a new way to assess both the electron density in the $\mathrm{N}^{+}$region and the total $\mathrm{N}$ abundance in an object. When there are few observations to warrant a detailed modeling approach, it may be necessary to use another approach which has been referred to as a semi-empirical method (hereafter SEM) (e.g. Aller 1984). We delineate a SEM scheme for doing this and apply it to observations for the H II region G333.6-0.2.

Electron Densities: There are several FIR diagnostics for $N_{e}$. These include the ratio of [O III] $52 / 88 \mu \mathrm{m},[\mathrm{S} \mathrm{III}] 19 / 33 \mu \mathrm{m}$, and [N II] $122 / 205 \mu \mathrm{m}$ - which provide $N_{e}\left[\mathrm{O}\right.$ III], $N_{e}$ [S III], and $N_{e}[\mathrm{~N}$ II] respectively. Each acts as an excellent discriminant of $N_{e}$ that is insensitive to $T_{e}$. The [N II] lines provide a new tool to examine low density, lower ionization gas. For conditions prevalent in most nebulae, these lines should readily show the effect of collisional deexcitation because their low critical densities $\left(N_{\text {crit }} \sim 40\right.$ and $260 \mathrm{~cm}^{-3}$ ) are much lower than for most lines used in nebular analyses. The $\mathrm{N}^{++} / \mathrm{N}^{+}$ratio and Stellar Effective Temperatures $T_{\text {eff }}$ : We provide a way to estimate $\mathrm{N}^{++} / \mathrm{N}^{+}$ based on the measurement of [N III] 57 and [N II] 122, $205 \mu$ m lines. We assume a 2-component model, with $\mathrm{N}^{+}$and $\mathrm{N}^{++}$zones, each having uniform density - using $N_{e}[\mathrm{~N} \mathrm{II}]$ and $N_{e}[\mathrm{~N} \mathrm{III]} \mathrm{(obtained} \mathrm{from}$ $N_{e}[\mathrm{O} I I I]$ and/or $\left.N_{e}\left[\mathrm{~S}_{\mathrm{III}}\right]\right)$. These yield the requisite volume emissivities and hence the $\mathrm{N}^{++} / \mathrm{N}^{+}$ ratio. Once $\mathrm{N}^{++} / \mathrm{N}^{+}$is found, we may estimate $T_{\text {eff }}$ by using curves relating these two, generated from H II region models (Rubin 1985). Because there is a fairly tight correlation (with a much smaller dependence on total number of ionizing photons/s and density), $T_{\text {eff }}$ may be inferred for an ionization bounded $\mathrm{H}$ II region as long as integrated fluxes have been measured. This technique has the advantage that it does not depend on prior knowledge of an abundance ratio.

The Total N Abundance $-(\mathrm{N} / \mathrm{H})$ : With $\mathrm{N}^{++} / \mathrm{N}^{+}$, we have, for the first time using FIR spectroscopy alone, the capability to assess the two dominant ionization states from the same element in $\mathrm{H}$ II regions or low ionization $\mathrm{PN}$ (with negligible $\mathrm{N}^{+3}$ and $\mathrm{N}^{0}$ ). To obtain $\mathrm{N} / \mathrm{H}$, an extinction corrected flux must be available for the same location/beam as used for the FIR fluxes for a generic hydrogen recombination line (or appropriate continuum surrogate). This derivation of $\mathrm{N} / \mathrm{H}$ requires neither that all of the flux in the lines be measured nor that the object be ionization bounded.

Application and Conclusions: The first discrete source in which both FIR [N II] $122,205 \mu \mathrm{m}$ lines have been measured is G333.6-0.2 - a luminous, obscured southern $\mathrm{H}$ II region, which has sufficiently low ionization that a substantial fraction of $\mathrm{N}$ is expected to be $\mathrm{N}^{+}$. ¿From measurements made with the Kuiper Airborne Observatory in a $45^{\prime \prime}$ beam with the facility cryogenic grating spectrometer, we obtain a preliminary $\log N_{e}[\mathrm{~N} \mathrm{II}]\left(\mathrm{cm}^{-3}\right) \sim 2.5$. This is significantly smaller than the value of $\log N_{e}[\mathrm{O}$ III $]$ and $\log N_{e}[\mathrm{~S}$ III $] \sim 3.6$. This difference may be due to the lower density in the $\mathrm{N}^{+}$ zone as well as to a biasing effect that causes lower $N_{e}$ values to be deduced for $N_{e}[\mathrm{~N} \mathrm{II}]$ (Rubin 1989). For G333.6, $\mathrm{N}^{++} / \mathrm{N}^{+}=13$. This large value cannot be used to infer $T_{\text {eff }}$ (except as an upper limit), because the $45^{\prime \prime}$ beam does not encompass all the [ $\mathrm{N} \mathrm{II}$ ] flux. The analysis for $\mathrm{N} / \mathrm{H}$ is still in progress; however, we estimate that $\mathrm{N} / \mathrm{H}$ increases merely by $\sim 7 \%$ due to the contribution of $\mathrm{N}^{+} / \mathrm{H}$ to a $45^{\prime \prime}$ beam centered on the source.

\section{References}

Aller, L.H. 1984, Physics of Thermal Gaseous Nebulae (Dordrecht: Reidel).

Rubin, R.H. 1985, $A p J S, \mathbf{5 7}, 349$.

Rubin, R.H. 1989, $A p J S, 69,897$. 